\title{
INDICATIONS FOR DIAMAGNETIC SHIFT IN THE GROUND STATE OF BORON IN SILICON
}

\author{
M. STöHR \\ Service National des Champs Intenses, CNRS, 38042 Grenoble, France \\ AND J.A. ChroboczeK \\ Centre National d'Études des Télécom., 38243 Meylan, France
}

\begin{abstract}
Indications for the observation of the diamagnetic shrinkage of the boron acceptor wave function (WF) in $\mathrm{Si}$ are reported. Uniaxial stress $(\boldsymbol{X})$ was used to split the ground state (GS) of the boron acceptor into two energy levels with spatially complementary WF. The magnetic field selectively induces a shrinking of one of the two WF, depending on whether it is applied parallel or perpendicular to the $\boldsymbol{X}$ axis. As a result, the hopping transitions between lower and higher energy levels are redistributed, leading to significant changes in the activation energy $\varepsilon_{3}$. This effect was borne out by experiment.
\end{abstract}

PACS numbers: 71.55.Ht, 71.70.-d, 72.20.-i

\section{Introduction}

The aim of this work was to find an indication for the existence of magnetically induced shrinkage of the impurity wave function of the boron impurity in silicon, similar to that observed previously in Ge:Ga [1]. However, the main effect of the magnetic field on shallow acceptor states in $\mathrm{Si}$ is the linear splitting of GS. Combining the effects of $\boldsymbol{X}$ and the magnetic field $\boldsymbol{B}$ one can induce crossing of the levels of the GS components which gives a non-monotonic behaviour of the piezomagnetoresistance (PMR) in the low $\boldsymbol{X}$ region, with a minimum corresponding to the stress at which the levels cross. This effect has been found in measurements on Si:B single crystals [2] and is confirmed in the present work on Si:B thin films. The film bending technique allowed the investigation of the negative stress region and experiments with the same sample at both $B \| X$ and $B \perp X$. Comparison of the PMR data obtained in both configurations provides indications of the diamagnetic shrinkage of the $\Gamma_{6}$ and $\Gamma_{7}$ wave functions, respectively. The shrinkage is related to the diamagnetic shifts of the GS components that are probably too small to be detected directly by spectroscopic means. 


\section{Experiment}

Transverse piezoresistivity measurements were carried out on narrow $(L=$ $13 \mathrm{~mm} / 20 \mathrm{~mm}, W=5 \mathrm{~mm} / 6 \mathrm{~mm}, t=0.3 \mathrm{~mm} / 0.5 \mathrm{~mm})$, [110] oriented slabs, cut from three different (111) Si wafers, with the MBE Si:B active layer of the thickness $d=1 \mathrm{~mm}$ and $4 \mathrm{~mm}$. The concentrations ranged from $2 \times 10^{17}$ to $4 \times 10^{17} \mathrm{~cm}^{-3}$ and the samples had both a low and medium range compensation. The contacts were made of the $\mathrm{Ga} / \mathrm{Al}$ eutectic, formed directly on the Si surface [3]. We used both four-point contact measurements and measurements on lithographically etched Hall bars, with the same results.

Samples' resistance $(R<10 \mathrm{G} \Omega)$ was measured with a remotely controlled, four-probe electrometric circuit with triaxial leads, partially described in Ref. [4].

Uniform uniaxial stresses $(-0.3 \mathrm{GPa}<X<0.3 \mathrm{GPa}, X\|[110] ; \boldsymbol{J}\|[112])$ were obtained in the active layer by substrate bending [5]. The stress was calculated using Bernoulli's formula for the bent beam problem:

$$
S(x)=(2 / 3) E t(L-x) / L^{3} z(L)
$$

where $E$ is the Young's modulus, $t$ - thickness of the substrate, $L-$ free length of the film, $x$ - distance of contacts from fixed end of film, and $z(L)$ - displacement of the film at its free end. A magnetic field was applied by a superconducting magnet $(B<17 \mathrm{~T}, \boldsymbol{B} \|[110]$ and $\boldsymbol{B} \|[111])$ and the temperatures $T$ were controlled in a continuous He flow cryostat. The interpretation of our results required the knowledge of the activation energy $\varepsilon_{3}$ and the preexponential $\rho_{3}$, defined by $\rho=\rho_{3} \exp \left(\varepsilon_{3} / k T\right)$. Therefore, the measurements were performed at two distinct temperatures, giving good results compared to full Arrhenius plot measurements.

\section{Results}

We present our results of the experimentally obtained activation energy $\varepsilon_{3}$ in Figs. 1 and 2. $\varepsilon_{3}(X, B=0)$ is a M-shaped function which is centred at $X=0$. For $B>0, B \| X$ the central minimum shifts from $X=0$ to some low and positive stress value. The maximum at $X>0$ weakens, whereas the maximum at $X<0$ is enhanced. The opposite is true for $B \perp X$, but no shift of the minimum occurs.

\section{Discussion}

Interpreting our results we use the basic notion of the percolation theory that the resistance of the crystal is proportional to the critical impedance at percolation, $Z_{\mathrm{c}}$. The latter is inversely proportional to a sum of transition probability terms, taking into account all the four components of the GS. Each term is a product of the square of the overlap of wave function components (overlap term, OT), centred at neighbouring acceptor sites, and a statistical term (ST), which depends on phonon and GS component occupation numbers.

The boron acceptor state has two complementary wave function components: slightly allongated spheres for the $\pm 1 / 2$ components and flattened spheres 

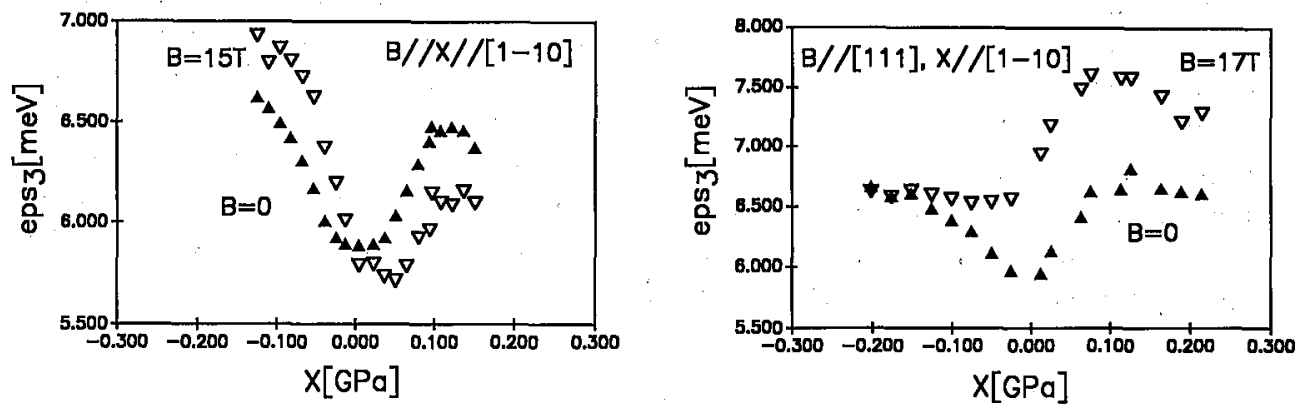

Fig. 1. The activation energy for a low compensated sample with $N_{\mathrm{B}}=2 \times 10^{17} \mathrm{~cm}^{-3}$ at a zero and a high magnetic field strength for $B \| X$.

Fig. 2. The activation energy for $B \perp X$. Sample characteristic as in Fig. 1.

for $\pm 3 / 2$ components [6]. When stress is applied, its direction becomes the symmetry axis of the wave function components and the states become separated in energy. Due to the splitting of the GS, two of the wave function components $( \pm 1 / 2$ for $X>0$ and $\pm 3 / 2$ for $X<0$ ) are more populated and the ST is appreciable only for the transitions between the (hole) lower energy components. The OT is large for the transitions within the spatial angle where the corresponding wave function components are more extended. At a spatial angle, where the higher energy wave function components are more extended, the appropriate OT can over-compensate the small ST and transitions via the higher energy components are activated (bypass effect). The activation energy $\varepsilon_{3}$ increases, since percolation includes now the transitions which are enhanced by the energy splitting of the GS. However, when the separation in energy becomes sufficiently large, the bypass effect will no longer work and transitions into any spatial angle will take place between the lower energy components alone and $\varepsilon_{3}$ will decrease again. Hence, $\varepsilon_{3}(X)$ shows maxima at some positive and at some approximately equal, but negative $X$ value. The magnetic field induces basically a linear correction to the acceptor state components energies. Simultaneous action of both $\boldsymbol{X}$ and $\boldsymbol{B}$, leads to a crossing of $\pm 1 / 2$ and $+3 / 2$ levels and a quasi-degenerate acceptor state at some low $X$ value. This value increases with increasing $B$. At the crossing point the total wave function is similar to that at $\boldsymbol{X}=0, \boldsymbol{B}=0$ and the total resistivity presents a minimum. This minimum was found for $\boldsymbol{B}\|\boldsymbol{X}\|[110]$ at approximately the same position as it has been found for $B\|X\|$ [100] [2]. This finding is accounted for by the calculation of the $X$ splitting of GS (cf. p. 1356 in Ref. [7]) and of the $g$-factors of its components [8]. For $B \perp X$ no energy splitting of the GS is available and we cannot interpret the results at this point in a qualitative manner. However the lack of the shifts can be traced to the fact that the $g$-factors of the GS components are smaller for $\boldsymbol{B} \perp \boldsymbol{X}$ than for $\boldsymbol{B} \| \boldsymbol{X}$.

We explain the increase of the maximum in $\varepsilon_{3}$ at $X>0, B>0$ for $B \perp \boldsymbol{X}$ and at $X<0, B>0$, for $B \| \boldsymbol{X}$, by the presence of a magnetically-induced small correction of the wave function (diamagnetic shrinking). The wave function 
shrinks in the plane perpendicular to $B$. For $B \| X$, this affects more the $\pm 3 / 2$ wave function components than the $\pm 1 / 2$ components. Transitions by the $\pm 3 / 2$ components are partially suppressed. At $X<0$ the bypass effect (by the excited $\pm 1 / 2$ states) is enhanced and the maximum in $\varepsilon_{3}(X)$ increases. At $X>0$ the bypass effect (by the $+3 / 2$ component) is reduced and the maximum of $\varepsilon_{3}(X)$ flattens. For $B \perp X$ the shrinking affects more the $\pm 1 / 2$ wave function components than the $\pm 3 / 2$ components and all the magnetically-induced changes of $\varepsilon_{3}(X)$ correspond to those of $\varepsilon_{3}(-X)$ for $B \| X$.

\section{Conclusions}

We measured the PMR of Si:B film samples, cut from three different wafers and having various acceptor concentrations and compensations. The measurements were done at $B \| \boldsymbol{X}$ and $\boldsymbol{B} \perp \boldsymbol{X}$, using the film bending method which allowed application of both positive and negative stress $(|X|<0.3 \mathrm{GPa})$ on the same sample. Our results could be qualitatively explained, using a four component model of the acceptor state and percolation theory arguments. Indications are there for the diamagnetic shift of the boron acceptor state in Si.

\section{Acknowledgements}

The samples used in this work have been fabricated at the CNET Meylan and we thank Dr. Arnaud d'Avitaya and Mr. Y. Campidelli for their preparation. We are grateful to Mr. Y. Gamberini for his excellent work on sample slicing and orienting. We acknowledge the EEC for financial support to our research group (Prof. J.C. Portal).

\section{References}

[1] J.A. Chroboczek, H. Fritzsche, C.-L. Jiang, M. Pollak, R.L. Wild, Phil. Mag. B 44,685 (1981).

[2] M. Stöhr, P. Janiszewski, J.A. Chroboczek, Mater. Sci. Forum 65-66, 241 (1990).

[3] J.A. Chroboczek, Arnaud d'Aritaya, 1986 Patent No 8616030 (France) Procédé de formation de contacts ohmiques sur du silicium.

[4] J.A. Chroboczek, J. Link, J. Phys. E: Sci. Instrum. 18, 56 (1985).

[5] J.A. Chroboczek, A. Briggs, Inst. Phys. Conf. Ser. 95, 143 (1989).

[6] R. Buczko, J.A. Chroboczek, Phil. Mag. B 50, 429 (1984).

[7] A.K. Ramdas, S. Rodriguez, Rep. Prog. Phys. 44, 1297 (1981).

[8] F. Merlet, B. Pajot, Ph. Arcas, A.-M. Jean-Louis, Phys. Rev. B 12, 3297 (1975).

[9] G. Feher, J.C. Hensel, E.A. Gere, Phys. Rev. Lett. 50, 429 (1960). 